\title{
A Whole-Genome Scan to Map Quantitative Trait Loci for Conformation and Functional Traits in Canadian Holstein Bulls
}

\author{
D. Kolbehdari, Z. Wang, ${ }^{1}$ J. R. Grant, B. Murdoch, A. Prasad, Z. Xiu, E. Marques, \\ P. Stothard, and S. S. Moore \\ Department of Agricultural, Food and Nutritional Science, University of Alberta, Edmonton, Alberta, Canada, T6G 2P5
}

\section{ABSTRACT}

Genetic improvement of livestock populations can be achieved through detection and mapping of genetic markers linked to quantitative trait loci (QTL). With the completion of the bovine genome sequence assembly, single nucleotide polymorphism (SNP) assays spanning the whole bovine genome and research work on large-scale identification, validation, and analysis of genotypic variation in cattle has become possible. A total of 462 Canadian Holstein Bulls were used to test the association between SNP and QTL. Single locus linkage disequilibrium regression model was implemented to perform a whole genome scan to identify and map QTL affecting conformation and functional traits. One thousand five hundred thirty-six SNP markers from introns and exons of potential QTL regions for economically important traits across the bovine genome were selected for association analysis. A total of 45 and 151 SNP were found to be associated with 17 conformation and functional traits at a genome- and chromosome-wise significance level, respectively. Among the 196 significant SNP, 169 of them are newly detected in this study, whereas 27 of them have been reported in previous literature and 161 of these were located in genes and are worth further investigating to potentially identify the causative mutations underlying the QTL. The single locus linkage disequilibrium regression method using SNP marker genotypes has proven to be a successful methodology for detecting and mapping QTL in dairy cattle populations.

Key words: genome scan, single nucleotide polymorphism, quantitative trait loci detection, linkage disequilibrium mapping

\section{INTRODUCTION}

Recent advances in molecular biotechnology that facilitate incorporation of molecular information into the

Received August 7, 2007.

Accepted March 10, 2008.

${ }^{1}$ Corresponding author: zhiquan@ualberta.ca traditional genetic evaluation models have enabled the identification and characterization of genes that contribute to genetic variation in economically important quantitative traits, hence leading to improvements in selection accuracies in livestock populations. Fernando and Grossman (1989) presented methodology for the application of BLUP to marker-assisted selection in which QTL alleles are considered random in the context of the mixed model terminology. Because DNA extraction is not restricted by age or sex, molecular genetics can alleviate some of the limitations of quantitative genetic selection (Dekkers and Hospital, 2002).

Since the initiation of whole genome scans in dairy cattle by Georges et al. (1995), several genome scans and QTL mapping projects have been undertaken to identify the genomic regions harboring genes that underlie phenotypic variation of production and conformation traits in dairy cattle (Smaragdov et al., 2006). The QTL that mapped to regions containing likely candidate genes are of special interest. Several QTL and candidate genes for milk production, reproduction, functional, and conformation traits have been identified in previous studies on Bos taurus autosomes (BTA) 1, 2, 3, 4, 5, 6, 7, 9, 14, 19, 20, 23, 26, 27, and 29. Some of these QTL were detected and mapped in numerous studies (Boichard et al., 2003; Ashwell et al., 2005; Schnabel et al., 2005).

Single nucleotide polymorphisms are the most abundant form of DNA variation in the genome (Snelling et al., 2005), and their preference is growing over other types of genetic markers due to lower mutation rate and the ease of genotyping (Hinds et al., 2005). Single nucleotide polymorphisms have been used for the detection and localization of QTL for complex traits in many species (Daw et al., 2005). In addition, the availability of the bovine genome sequence assembly has accelerated the ability to associate specific genome variation with phenotypic variation across the bovine genome, which is the ultimate goal of many QTL identification and mapping projects. In cattle, a 7.1 $\times$ sequence assembly has been produced with accompanying information on over 2,300,000 SNP genome-wide (Bovine Genome 
Project: http://www.hgsc.bcm.tmc.edu/projects/bovine). A set of SNP assays spanning the whole bovine genome becomes possible after mining this data, which helps accelerate the research work on large-scale identification, validation, and analysis of genotypic variation in cattle.

Several statistical approaches have been developed for whole genome scans and QTL mapping projects. The least squares model based on regression of phenotype on marker genotypes or haplotypes and the random effects models based on identity by descent approaches (George et al., 2000; Kolbehdari et al., 2005; Gautier et al., 2006) are commonly used. High-density SNP marker genotypes have increased the feasibility of QTL detection and mapping using historical population-wide linkage disequilibrium (LD), which requires a marker allele to be in LD with the QTL allele across the entire population. Linkage disequilibrium can be a result of migration, mutation, selection, small finite population size, or other genetic events that the population experiences. In livestock populations, finite population size is generally implicated as the key cause of $\mathrm{LD}$, because effective population sizes for most livestock population are relatively small (Meuwissen and Goddard, 2000). Indeed, extensive LD has been observed in dairy cattle, sheep, and pig populations in previous studies (Farnir et al., 2000; Dekkers and Hospital, 2002).

The LD method regresses the phenotypes of the quantitative trait on the marker genotypes. Regression on single SNP marker genotypes does not require knowledge of SNP position and linkage phase and is therefore easier to implement. Studies have shown that a single marker test based on the LD regression model provided similar or greater power than a haplotype-based and an identity by descent-based model (Grapes et al., 2004; Zhao et al., 2007), and QTL could be detected and mapped by the LD regression method. Further, this method offers a greater flexibility to include dominance and epistatic effects. In addition, the random polygenic effects accounting for relationships among individuals could be added to this model. The objective of this study was to perform a whole genome scan to identify and map QTL affecting conformation and functional traits using the LD regression method and SNP genotype markers in the Canadian Holstein population.

\section{MATERIALS AND METHODS}

\section{Genotyping Assay Design and Genotyping Platform}

A total of 1,536 SNP markers were selected to represent introns and exons of potential candidate genes across the bovine genome sequence assembly (Btau_2.0). A subset of bovine SNP already characterized by the Alberta Bovine Genomics Laboratory was combined into a single multiplex assay of 1,536 SNP for analysis on the Illumina Beadstation 500G SNP genotyping platform (Oliphant et al., 2002). Among these SNP, 139 poorly amplified and 56 monomorphic SNP during the genotyping were removed from the analysis. The genotyping assay was designed based on bovine genome sequence assembly version 2 (Btau_2.0). In the original design, these SNP were selected to be distributed as evenly as possible based on the physical locations on the bovine genome sequence assembly (Btau_2.0). The SNP known to be in putative candidate genes for milk production and confirmation traits were strategically added to the assay. However, after the assay was manufactured, an updated version of the bovine genome sequence assembly (Btau_3.1) was released. Chromosome assignments and SNP positions were recalculated based on the updated version of bovine genome sequence assembly.

A local database was developed that contains over 1.8 million bovine SNP and almost 30,000 genes gathered from the databases at the National Center for Biotechnology Information (http://www.ncbi.nlm.nih. gov/genome/seq/BlastGen/BlastGen). Querying the database provided details on each SNP, including its location and functional class. The SNP that are present in a gene locus can have one of the following National Center for Biotechnology Information-defined functional classes: locus-region, coding, coding-synonymous, coding-nonsynonymous, mRNA-UTR, intron, and splice-site. For SNP not located in a gene, the nearest gene was determined by querying the database for a list of genes on the same chromosome as the SNP, and the gene closest to the location of the SNP was identified.

\section{Animal Resource and Description of Phenotypic Data}

A total of 462 Canadian Holstein bulls from Semex Canada (Guelph, Ontario, Canada), 319 of them originating from 10 core sire families, were used in the study. The DNA was extracted from bull semen and genotyped. Complete general pedigree information along with the most updated bull EBV (November 2006) were provided by the Canadian Dairy Network (Guelph, Ontario, Canada). The SNP genotypes were used to test the association with measured economically important traits including major type traits (overall conformation, mammary system, feet and legs, dairy strength, rump), 6 descriptive type traits (udder texture, median suspensory, foot angle, bone quality, stature, angularity), and 6 functional traits (herd life, daughter fertility, milking speed, milking temperament, calving ease, and maternal calving ease). Bull genotypes of the studied traits 
Table 1. Descriptive statistics of the conformations and functional traits for the studied 462 bulls

\begin{tabular}{lcccc}
\hline Traits & Mean & SD & Minimum & Maximum \\
\hline Conformation & 3.04 & 5.56 & -17 & 19 \\
Mammary system & 2.88 & 5.79 & -18 & 19 \\
Feet and leg & 1.73 & 5.06 & -17 & 18 \\
Dairy strength & 1.78 & 5.16 & -14 & 17 \\
Rump & 0.99 & 4.93 & -20 & 21 \\
Udder texture & 1.85 & 4.68 & -16 & 27 \\
Median suspensory & 2.39 & 5.36 & -17 & 16 \\
Foot angle & 0.39 & 5.21 & -14 & 18 \\
Bone quality & 0.86 & 4.95 & -19 & 15 \\
Stature & 1.49 & 5.23 & -18 & 16 \\
Angularity & 2.51 & 4.99 & -18 & 3.65 \\
Herd life & 3.08 & 0.24 & 2.49 & 74 \\
Daughter fertility & 65.56 & 3.37 & 57 & 95 \\
Milking speed & 85.51 & 4.14 & 70 & 96 \\
Milking temperament & 89.59 & 3.31 & 70 & 96 \\
Calving ease & 84.4 & 5.1 & 56 & 96 \\
Maternal calving ease & 86.11 & 5.37 & 62 & \\
\hline
\end{tabular}

are preferred for gene association studies over their raw phenotypic records, because the EBV of each bull is based on the records of many daughters and is therefore a much more accurate estimate of the genetic potential of each animal than a single cow phenotype. The descriptive statistics of these traits that were estimated from the 462 bulls that have been genotyped in current study are given in Table 1.

The definitions of the above traits are available at Canadian Dairy Network (http://www.cdn.ca/articles.php). Daughter fertility is a new fertility trait, which was provided by the Canadian Dairy Network for genetic evaluation of dairy sires and calculation of bull proofs for 4 different traits related to the fertility of dairy cattle. These include age at first insemination for heifers, 56-d nonreturn rate (NRR) in heifers, the interval from calving to first insemination within each lactation for cows, and the 56-d nonreturn rate in cows. The ability of an animal to remain productive in a dairy herd is of interest and is profitable for dairy cattle producers. Genetic evaluation values of herd life of bulls are calculated based on actual survival data of their daughters.

\section{Statistical Analysis}

To test the association between SNP and the QTL, a single locus LD regression model was implemented in this study. The markers were assumed to be in $\mathrm{LD}$ with the QTL over the entire genome. The simple regression model based on LD has been shown, using simulated data, to have an acceptable level of power and accuracy for fine mapping QTL (Grapes et al., 2004; Zhao et al., 2007). The following mixed linear LD regression model was fitted in this study by using the ASReml package (Gilmour et al., 2006):

$$
\mathbf{y}=\mathbf{X} \mathbf{b}+\mathbf{Z} \mathbf{a}+\mathbf{e}
$$

where $\mathbf{y}=$ the vector of phenotypes (EBV); $\mathbf{X}=$ the design matrix; $\mathbf{b}=$ the vector of coefficients of the regression on recoded SNP genotypes; $\mathbf{Z}=$ the incidence matrix for animal effects; $\mathbf{a}=$ a vector of the polygenic animal effects; and $\mathbf{e}=$ the vector of residuals.

The QTL allele substitution effects and additive genetic effects were evaluated in this model. The design matrix, $\mathrm{X}$, was coded as 2, 1, 0 for the SNP genotypes 1-1, 1-2, and 2-2, respectively. The F-statistic, type I error ( $P$-value), and allele substitution effects were estimated for all SNP genotypes across the whole genome.

A major issue in multiple testing is the proper setting of significance thresholds. A useful statistic is false discovery rate (FDR), which is the expected proportion of falsely detected QTL (Benjamini and Hochberg, 1995). The FDR takes into account the number of tests that are performed as well as how significant one test is relative to the others in multiple comparison procedures. Family-wise error rate controls the probability of committing any type I error in families of comparisons. The FDR was used to establish the statistical significance critical value in this study. Two levels of significant controls were used in this study based on genome-wise and chromosome-wise type I errors, which were computed for all SNP (Benjamini and Hochberg, 1995; Fernando et al., 2004). The genome-wise level is a very conservative approach for a large number of markers in a whole genome scan study. Therefore, if SNP exceed the genome-wise type I error, they should be highly associated with the trait.

The FDR was calculated for a QTL on a genome or a chromosome-wise level by assuming $n$ number of tests performed on a genome or a chromosome, and the $P$ - 
Table 2. Single nucleotide polymorphism (SNP) number and the average intervals between $\operatorname{SNP}\left(\mathrm{kbp}^{1}\right)$ by chromosome

\begin{tabular}{lcc}
\hline BTA $^{2}$ & Frequency & Intervals $(\mathrm{kbp})$ \\
\hline 1 & 64 & $2,193.3$ \\
2 & 64 & $1,935.3$ \\
3 & 78 & $1,492.4$ \\
4 & 75 & $1,424.5$ \\
5 & 59 & $1,828.6$ \\
6 & 40 & $2,749.2$ \\
7 & 71 & $1,392.8$ \\
8 & 36 & $2,788.1$ \\
9 & 33 & $2,656.7$ \\
10 & 44 & $2,058.6$ \\
11 & 56 & $1,722.1$ \\
12 & 14 & $5,530.7$ \\
13 & 54 & $1,452.9$ \\
14 & 27 & 2,690 \\
15 & 67 & $1,115.6$ \\
16 & 48 & $1,432.7$ \\
17 & 39 & $1,809.7$ \\
18 & 44 & $1,380.9$ \\
19 & 64 & 991.7 \\
20 & 29 & $2,223.1$ \\
21 & 32 & $1,950.3$ \\
22 & 42 & $1,425.7$ \\
23 & 44 & $1,075.7$ \\
24 & 19 & $2,877.4$ \\
25 & 40 & $1,058.5$ \\
26 & 41 & $1,142.9$ \\
27 & 16 & $2,123.7$ \\
28 & 34 & $1,135.6$ \\
29 & 67 & 668.9 \\
Total & 141 & \\
\hline & &
\end{tabular}

${ }^{1} \mathrm{kbp}=$ kilobase pairs.

${ }^{2} \mathrm{BTA}=$ Bos taurus autosome.

values were ranked from lowest to highest. The following equation was used to calculate the FDR:

$$
F D R=\frac{n \times P(k)}{k}
$$

where $k=$ the individual relative test position.

\section{RESULTS AND DISCUSSION}

\section{SNP Analysis}

The SNP used in this study were distributed throughout the bovine genome. The total number of SNP on each chromosome varied from 14 on BTA 12 to 78 on BTA 3. The average interval between SNP on different chromosomes varied from 668.9 kilobase pairs on BTA 29 to 5,530.7 kilobase pairs on BTA 12. The X chromosome was not included in this study. The average SNP polymorphism information content was 0.287 ( 0 to 0.375 ), and the average observed SNP heterozygosity was 0.389 . The details of the number of SNP per chromosome and their average interval in kilobase pairs per chromosome are given in Table 2 . The genotyping assay was originally designed based on Bovine Genome Sequence Assembly version 2 (Btau_2.0). New chromosome assignments and SNP positions were calculated based on the updated version of bovine genome sequence assembly (Btau_3.1). This recalculation resulted in a less even distribution of SNP than originally intended.

\section{Major Type Traits}

The whole genome scan to map QTL for major type traits including overall conformation, mammary system, feet and legs, dairy strength, and overall rump identified 24 SNP significantly associated with these traits at the genome-wise level and 36 at the chromosome-wise level $(P<0.05)$. The details of the SNP showing association with these traits at the genome and chromosome significance level, including their heterozygosity, position, F-test statistic value, allele substitution effects, and $P$-value are shown in Tables 3 to 7 .

Five SNP had significant effects on overall conformation at the chromosome-wise level $(P<0.05)$ on BTA 4 , 18, 21, 23, and 29 (Table 3). A larger F-test statistic showed strong evidence of association between these chromosome regions of the bovine genome with overall conformation. For instance, SNP rs41634501 on BTA $23(23,107,013 \mathrm{bp})$ had a F-test statistic of 14.59 and showed significant association with overall conformation at the chromosome-wise level $(P<0.01)$ with allele substitution effects of 1.58 . These 5 significant regions may contain QTL responsible for overall conformation, which have not been reported previously. Of the 5 sig-

Table 3. Significant genome- and chromosome-wise single nucleotide polymorphisms (SNP) with conformation

\begin{tabular}{|c|c|c|c|c|c|c|c|c|}
\hline SNP NCBI ${ }^{1}$ ID & $\mathrm{BTA}^{2}$ & Position (bp) & Heterozygosity & F-test & Effects & $P$-value & Functional class & Gene name \\
\hline rs41636734 & 18 & $53,743,293$ & 0.48 & 11.62 & 1.24 & $0.00037^{*}$ & intron & CD37 \\
\hline rs41565403 & 21 & $33,224,592$ & 0.28 & 7.78 & 1.54 & $0.003^{*}$ & coding-synonymous & STRA6 \\
\hline rs 29024010 & 29 & $30,954,390$ & 0.31 & 10.67 & 1.84 & $0.00062^{*}$ & intron & HNT \\
\hline
\end{tabular}

${ }^{1} \mathrm{NCBI}=$ National Center for Biotechnology Information.

${ }^{2} \mathrm{BTA}=$ Bos taurus autosome.

*Significant chromosome-wise $(P<0.05)$; **significant chromosome-wise $(P<0.01)$. 
Table 4. Significant chromosome-wise single nucleotide polymorphisms (SNP) with mammary system

\begin{tabular}{|c|c|c|c|c|c|c|c|c|}
\hline $\mathrm{SNP} \mathrm{NCBI}^{2} \mathrm{ID}$ & $\mathrm{BTA}^{2}$ & Position (bp) & Heterozygosity & F-test & Effects & $P$-value & Functional class & Gene name \\
\hline rs41652648 & 5 & $81,674,580$ & 0.47 & 11.50 & -1.46 & $0.0004^{*}$ & intron & ITPR2 \\
\hline rs41636734 & 18 & $53,743,293$ & 0.48 & 12.95 & 1.41 & $0.00018^{* *}$ & intron & CD37 \\
\hline rs41653440 & 28 & $25,280,138$ & 0.47 & 9.45 & 1.33 & $0.0012^{*}$ & intron & PSAP \\
\hline
\end{tabular}

${ }^{1} \mathrm{NCBI}=$ National Center for Biotechnology Information.

${ }^{2} \mathrm{BTA}=$ Bos taurus autosome.

*Significant chromosome-wise $(P<0.05)$; **significant chromosome-wise $(P<0.01)$.

nificant SNP, SNP rs41636734 and SNP rs29024010 are located in the introns of predicted genes (CD37 and HNT), SNP rs41565403 is a synonymous coding SNP, whereas the remaining 2 SNP are not located in genes (Table 3). It has been found that SNP rs41591551 also shows significant association with median suspensory, whereas SNP rs41636734 also shows significant association with rump and mammary system.

Two SNP had significant effects on the mammary system at the chromosome-wise level at $P<0.05$ on BTA 5 and 28 (Table 4), whereas SNP rs41636734 on BTA $18(53,743,293 \mathrm{bp})$ had a significant effect at the genome-wise level at $P<0.01$. These SNP are located in introns of predicted genes (ITPR2, CD37, and PSAP). Of the 3 significant regions found in the current study, 2 of them $(53,743,293$ and $25,280,138$ bp on BTA 18 and BTA 28) are in regions reported in a previous study by Ashwell et al. (2005). It has been found that SNP rs41636734 also shows significant association with conformation and rump.
Ten SNP were found to have a significant association with feet and legs at the genome-wise and 9 at the chromosome-wise level $(P<0.05)$ on BTA $1,2,4,10$, $13,14,21,22,23$, and 27 (Table 5). Ten of these significant SNP are located in the introns of predicted genes, 3 of them are synonymous coding SNP, and 6 of them are not located in genes (Table 5). Two of the 19 regions, BTA $13(64,863,304 \mathrm{bp})$ and BTA $14(40,196,763 \mathrm{bp})$, have been reported in a previous study to be associated with feet and legs by Ashwell et al. (2005). Two of these SNP (rs41645645 and rs43710950) show significant association with foot angle. Five of these SNP (rs41645645, rs41632254, rs41603160, rs41645547, rs41643866) also show significant association with bone quality. Three of these SNP (rs41636945, rs41632254, rs41634501) also shows significant association with median suspensory. It has been found that SNP rs41643866 also shows significant association with herd life, whereas SNP rs41647383 also shows significant association with milking speed.

Table 5. Significant genome- and chromosome-wise single nucleotide polymorphisms (SNP) with feet and legs

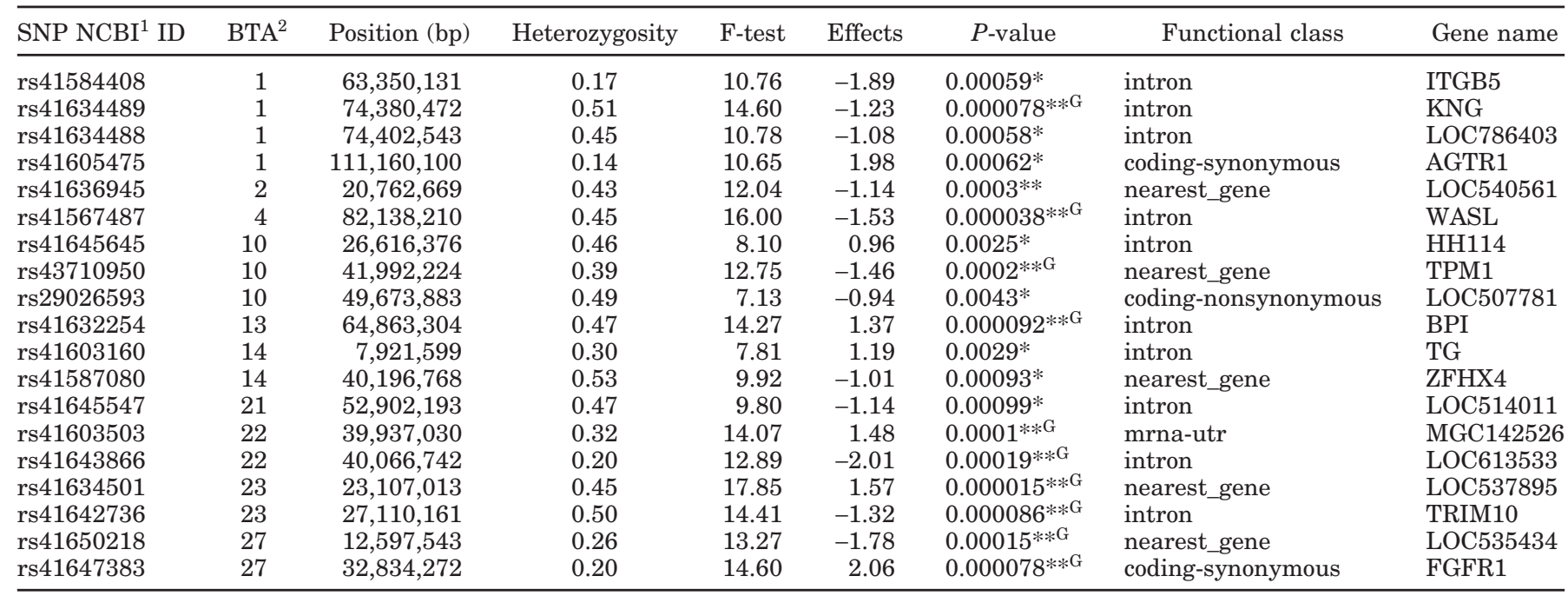

${ }^{1} \mathrm{NCBI}=$ National Center for Biotechnology Information.

${ }^{2} \mathrm{BTA}=$ Bos taurus autosome.

*Significant chromosome-wise $(P<0.05)$; **significant chromosome-wise $(P<0.01)$.

${ }^{\mathrm{G}}$ Significant genome-wise $(P<0.05)$. 
Table 6. Significant genome- and chromosome-wise single nucleotide polymorphisms (SNP) with dairy strength

\begin{tabular}{|c|c|c|c|c|c|c|c|c|}
\hline SNP $\mathrm{NCBI}^{1} \mathrm{ID}$ & $\mathrm{BTA}^{2}$ & Position (bp) & Heterozygosity & F-test & Effects & $P$-value & Functional class & Gene name \\
\hline rs41601701 & 3 & $98,500,067$ & 0.08 & 11.03 & 2.69 & $0.00051^{* *}$ & coding-synonymous & MGC139728 \\
\hline rs41604534 & 5 & $29,067,052$ & 0.55 & 7.32 & 1.00 & $0.0039 *$ & intron & KRT5 \\
\hline rs29012216 & 5 & $40,289,953$ & 0.46 & 12.18 & 1.38 & $0.00028 * * \mathrm{G}$ & intron & BSM \\
\hline rs41604564 & 5 & $58,756,658$ & 0.33 & 12.61 & -1.38 & $0.00022 * * \mathrm{G}$ & nearest_gene & LTA4H \\
\hline rs29014633 & 5 & $74,016,631$ & 0.44 & 16.91 & 1.58 & $0.000024^{* * \mathrm{G}}$ & intron & CACNG2 \\
\hline rs41609478 & 7 & $37,343,116$ & 0.50 & 16.77 & 1.48 & $0.000025^{* * \mathrm{G}}$ & nearest_gene & LOC538639 \\
\hline rs41591943 & 7 & $39,956,874$ & 0.37 & 13.39 & 1.51 & $0.00015^{* * \mathrm{G}}$ & nearest_gene & LOC527275 \\
\hline rs41661265 & 7 & $50,312,323$ & 0.46 & 15.93 & -1.36 & $0.000039 * * \mathrm{G}$ & coding-synonymous & MGC155209 \\
\hline rs41653025 & 10 & $49,942,146$ & 0.23 & 10.11 & -1.68 & $0.00084^{*}$ & coding-synonymous & LOC540856 \\
\hline rs41566192 & 13 & $43,058,415$ & 0.52 & 13.26 & -1.27 & $0.00016^{* * \mathrm{G}}$ & coding-nonsynonymous & MGC127374 \\
\hline rs 41602212 & 19 & $37,837,691$ & 0.46 & 16.91 & 1.53 & $0.000024 * * \mathrm{G}$ & intron & CACNA1G \\
\hline
\end{tabular}

${ }^{1} \mathrm{NCBI}=$ National Center for Biotechnology Information.

${ }^{2} \mathrm{BTA}=$ Bos taurus autosome.

*Significant chromosome-wise $(P<0.05)$; **significant chromosome-wise $(P<0.01)$.

${ }^{\mathrm{G}}$ Significant genome-wise $(P<0.05)$.

Eleven SNP were found to have a significant effect on dairy strength at the genome-wise and 9 at the chromosome-wise level $(P<0.05)$ on BTA 3, 5, 7, 10, 13, 19, 21,25 , and 26 (Table 6). Eight of these significant SNP are located in the introns of predicted genes, 7 of them are synonymous coding SNP, whereas 5 are not located in genes (Table 6). It has been found that SNP rs41604564 on BTA $5(58,756,658 \mathrm{bp})$ corresponds to a QTL that has been previously reported to have an effect on dairy strength by Ashwell et al. (2005), who also detected several QTL regions affecting dairy strength.
Only 1 of these regions on BTA 5 was in a similar region to those found in the current study (SNP rs41604564). There are several potential reasons for this inconsistency between the 2 studies. One cause could be the presence of different QTL in the 2 animal populations (1,404 bulls from 10 families of the North American Holstein-Friesian). Another could be a lack of informative markers in this study for the regions found by Ashwell et. al. (2005). Four of these SNP (rs41601701, rs41609478, rs41661265, rs41566192) also show significant association with angularity. It has been found

Table 7. Significant genome- and chromosome-wise single nucleotide polymorphisms (SNP) with overall rump

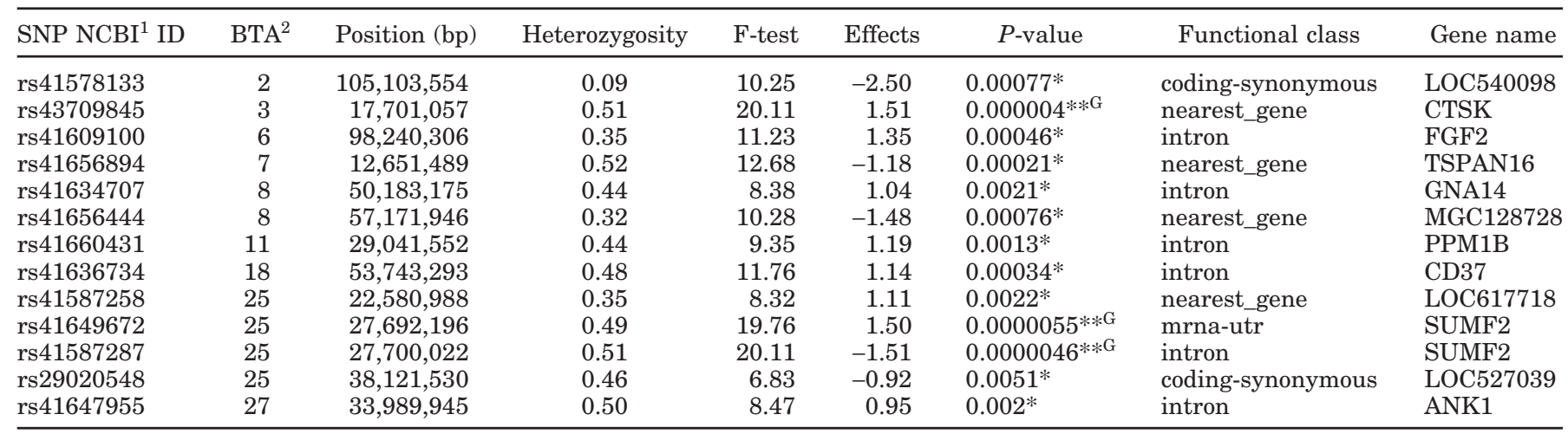

${ }^{1} \mathrm{NCBI}=$ National Center for Biotechnology Information.

${ }^{2} \mathrm{BTA}=$ Bos taurus autosome.

*Significant chromosome-wise $(P<0.05)$; **significant chromosome-wise $(P<0.01)$.

${ }^{\mathrm{G}}$ Significant genome-wise $(P<0.05)$. 
Table 8. Significant genome- and chromosome-wise single nucleotide polymorphisms (SNP) with udder texture

\begin{tabular}{|c|c|c|c|c|c|c|c|c|}
\hline SNP NCBI ${ }^{1}$ ID & $\mathrm{BTA}^{2}$ & Position (bp) & Heterozygosity & F-test & Effects & $P$-value & Functional class & Gene name \\
\hline rs41572038 & 3 & $57,607,378$ & 0.27 & 22.85 & 2.37 & $0.0000012^{* * \mathrm{G}}$ & intron & LEC2 \\
\hline rs41604605 & 5 & $9,208,135$ & 0.51 & 7.96 & 1.04 & $0.0027^{*}$ & intron & LOC780969 \\
\hline rs41655901 & 5 & $28,108,890$ & 0.47 & 9.18 & -1.09 & $0.0014^{*}$ & intron & GALNT6 \\
\hline rs41656703 & 5 & $32,429,000$ & 0.52 & 8.10 & -1.15 & $0.0025 *$ & nearest_gene & MGC127063 \\
\hline rs41591891 & 5 & $81,733,566$ & 0.44 & 12.27 & 1.53 & $0.00026^{*}$ & intron & ITPR2 \\
\hline rs41588786 & 25 & $3,434,609$ & 0.48 & 9.88 & 1.22 & $0.00095^{*}$ & nearest_gene & LOC505453 \\
\hline rs41650226 & 27 & $12,861,193$ & 0.35 & 8.58 & -1.32 & $0.0019 *$ & intron & IRF2 \\
\hline
\end{tabular}

${ }^{1} \mathrm{NCBI}=$ National Center for Biotechnology Information.

${ }^{2} \mathrm{BTA}=$ Bos taurus autosome.

*Significant chromosome-wise $(P<0.05)$; **significant chromosome-wise $(P<0.01)$.

${ }^{\mathrm{G}}$ Significant genome-wise $(P<0.05)$.

that SNP rs29014633 also shows significant association with angularity and stature, and SNP rs41602212 also shows significant association with angularity and milking temperament.

Three SNP were detected to have a significant effect on overall rump at the genome-wise and 10 at the chromosome-wise level $(P<0.05)$ on BTA $3,6,7,8,11,18$, 25 , and 27 (Table 7). Of these significant SNP, 6 are located in the introns of predicted genes, 2 are synonymous coding SNP, and 5 are not located in any genes (Table 7). These 13 significant regions have not been reported previously. Because there is no comparable result from the literature, further investigations are encouraged in the future. In addition, SNP rs41660431 also shows significant association with herd life and daughter fertility.

\section{Descriptive Type Traits}

The whole genome scan to map QTL for 6 descriptive type traits identified 13 SNP significantly associated with these traits at the genome-wise level and 57 at the chromosome-wise level $(P<0.05)$. The details of the SNP are shown in Tables 8 to 13, for udder texture, median suspensory, foot angle, bone quality, stature, and angularity, respectively.
One SNP had significant effects on udder texture at the genome-wise and 6 at the chromosome-wise levels $(P<0.05)$ on BTA $3,5,25$, and 27 (Table 8). It was found that SNP rs41572038 on BTA $3(57,607,378 \mathrm{bp})$ had a significant effect on udder texture at the genomewise level with an F-test statistic of 22.85 and allele substitution effects of 2.37. Of these significant SNP, 5 are located in the introns of predicted genes, and 2 of them are not located in genes (Table 8). The regions around SNP rs41591891 on BTA $5(81,733,566$ bp) has also been reported by Ashwell et al. (2005) for udder index at $119 \mathrm{cM}$. Two SNP (rs41588786, rs41650226) also show significant association with bone quality, whereas SNP rs41588786 was also significant with median suspensory. It has been found that SNP rs41656703 also shows significant association with dairy strength, whereas SNP rs41572038 also shows significant association with 2 traits stature, and herd life.

Nine SNP had significant effects on the median suspensory at the chromosome-wise level $(P<0.05)$ on BTA $2,4,5,13,22,23$, and 25 (Table 9). Two of these SNP (rs41655901 and rs41634501) on BTA 5 and BTA 23, respectively, have a significant effect on median suspensory at the chromosome-wise level $(P<0.01)$. Of the significant median suspensory SNP, 4 are located in

Table 9. Significant genome- and chromosome-wise single nucleotide polymorphisms (SNP) with median suspensory

\begin{tabular}{|c|c|c|c|c|c|c|c|c|}
\hline SNP NCBI ${ }^{1}$ ID & BTA $^{2}$ & Position (bp) & Heterozygosity & F-test & Effects & $P$-value & Functional class & Gene name \\
\hline rs41637504 & 2 & 555,621 & 0.45 & 8.98 & 1.01 & $0.0015^{*}$ & nearest_gene & LOC512912 \\
\hline rs41636945 & 2 & $20,762,669$ & 0.43 & 7.96 & -0.99 & $0.0027^{*}$ & nearest_gene & LOC540561 \\
\hline rs41591551 & 4 & $96,244,404$ & 0.36 & 11.22 & 1.31 & $0.00046^{*}$ & nearest_gene & KIAA1549 \\
\hline rs41632254 & 13 & $64,863,304$ & 0.47 & 9.95 & 1.21 & $0.00091^{* *}$ & intron & BPI \\
\hline rs41644471 & 22 & $46,449,737$ & 0.46 & 8.32 & -1.13 & $0.0022^{*}$ & intron & CACNA1D \\
\hline rs41634501 & 23 & $23,107,013$ & 0.45 & 13.22 & 1.43 & $0.00015^{* *}$ & nearest_gene & LOC537895 \\
\hline
\end{tabular}

${ }^{1} \mathrm{NCBI}=$ National Center for Biotechnology Information.

${ }^{2} \mathrm{BTA}=$ Bos taurus autosome.

*Significant chromosome-wise $(P<0.05)$; ${ }^{* *}$ significant chromosome-wise $(P<0.01)$. 
Table 10. Significant genome- and chromosome-wise single nucleotide polymorphisms (SNP) with foot angle

\begin{tabular}{|c|c|c|c|c|c|c|c|c|}
\hline SNP NCBI ${ }^{1}$ ID & $\mathrm{BTA}^{2}$ & Position (bp) & Heterozygosity & F-test & Effects & $P$-value & Functional class & Gene name \\
\hline rs41584408 & 1 & $63,350,131$ & 0.17 & 7.40 & -1.56 & $0.0037 *$ & intron & ITGB5 \\
\hline rs41567487 & 4 & $82,138,210$ & 0.45 & 14.67 & -1.46 & $0.000075^{* * \mathrm{G}}$ & intron & WASL \\
\hline rs41642656 & 5 & $102,927,637$ & 0.45 & 20.05 & -1.79 & $0.0000047 * * \mathrm{G}$ & intron & VWF \\
\hline rs41595440 & 7 & $57,368,652$ & 0.55 & 10.63 & -1.20 & $0.00063^{*}$ & coding-nonsynonymous & LOC509419 \\
\hline rs41645645 & 10 & $26,616,376$ & 0.46 & 7.99 & 0.94 & $0.0027^{*}$ & intron & HH114 \\
\hline rs43710950 & 10 & $41,992,224$ & 0.39 & 12.08 & -1.41 & $0.00029 *$ & nearest_gene & TPM1 \\
\hline rs41634818 & 16 & $65,875,535$ & 0.46 & 15.45 & 1.42 & $0.00005^{* * \mathrm{G}}$ & intron & KCNH1 \\
\hline
\end{tabular}

${ }^{1} \mathrm{NCBI}=$ National Center for Biotechnology Information.

${ }^{2} \mathrm{BTA}=$ Bos taurus autosome.

*Significant chromosome-wise $(P<0.05)$; **significant chromosome-wise $(P<0.01)$.

${ }^{\mathrm{G}}$ Significant genome-wise $(P<0.05)$.

the introns of predicted genes, and 5 of them are not located in genes (Table 9). Two of these regions (555,621 and $64,863,304 \mathrm{bp}$ ) on BTA 2 and BTA 13 have been reported for front udder attachment and udder index by Ashwell et al. (2005). These 2 regions on BTA 2 and BTA 13 are good candidate regions for further investigation of candidate QTL affecting median suspensory and udder attachment in dairy cattle. It has been found that SNP rs41632254 also shows significant association with feet and leg and bone quality, whereas SNP rs41634501 also shows significant association with overall conformation and feet and leg.
Three SNP had significant effects on foot angle at the genome-wise and 8 at the chromosome-wise levels $(P<0.05)$ on BTA $1,4,5,7,10$, and 16 (Table 10). Eight of these SNP are located in the introns of predicted genes, 2 of these SNP are synonymous coding SNP, whereas 1 is not located in any gene (Table 10). One of these regions, $(57,368,652 \mathrm{bp})$ on BTA 7 , has been reported for this trait at $83 \mathrm{cM}$ of this chromosome by Ashwell et al. (2005). This region of the bovine genome showed strong evidence of harboring QTL affecting foot angle in cattle. It has been found that SNP rs41653025 also shows significant association with dairy strength.

Table 11. Significant genome- and chromosome-wise single nucleotide polymorphisms (SNP) with bone quality

\begin{tabular}{|c|c|c|c|c|c|c|c|c|}
\hline $\mathrm{SNP} \mathrm{NCBI}^{1} \mathrm{ID}$ & $\mathrm{BTA}^{2}$ & Position (bp) & Heterozygosity & F-test & Effects & $P$-value & Functional class & Gene name \\
\hline rs41639301 & 1 & $114,963,599$ & 0.34 & 12.01 & 1.53 & $0.00031^{*}$ & coding-synonymous & LOC533642 \\
\hline rs29023607 & 3 & $1,043,852$ & 0.32 & 9.71 & 1.46 & $0.0011^{*}$ & intron & CD3Z \\
\hline rs41650242 & 5 & $74,090,417$ & 0.17 & 14.11 & 2.28 & $0.0001 * * \mathrm{G}$ & intron & CACNG2 \\
\hline rs41658737 & 7 & $10,335,915$ & 0.30 & 8.68 & 1.38 & $0.0018^{*}$ & intron & CACNA1A \\
\hline rs41588239 & 7 & $12,077,407$ & 0.45 & 9.92 & -1.24 & $0.00092 *$ & coding-synonymous & GADD45GIP1 \\
\hline rs41658022 & 7 & $35,844,869$ & 0.43 & 14.66 & 1.44 & $0.000075^{* * \mathrm{G}}$ & intron & SLC34A1 \\
\hline rs41603160 & 14 & $7,921,599$ & 0.30 & 12.85 & 1.58 & $0.00019 * * \mathrm{G}$ & intron & TG \\
\hline rs29010770 & 19 & $21,530,439$ & 0.49 & 10.79 & -1.08 & $0.00057 * *$ & coding-synonymous & RPA1 \\
\hline rs41600178 & 19 & $41,647,314$ & 0.13 & 10.00 & -2.03 & $0.00088^{*}$ & nearest_gene & GAS \\
\hline rs41581582 & 20 & $51,741,640$ & 0.20 & 7.81 & 1.53 & $0.0029 *$ & intron & FLJ20152 \\
\hline rs41643786 & 21 & $45,669,248$ & 0.36 & 7.76 & -1.19 & $0.003^{*}$ & coding-synonymous & MGC133693 \\
\hline rs41645547 & 21 & $52,902,193$ & 0.47 & 9.28 & -1.16 & $0.0013^{*}$ & intron & LOC514011 \\
\hline
\end{tabular}

${ }^{1} \mathrm{NCBI}=$ National Center for Biotechnology Information.

${ }^{2} \mathrm{BTA}=$ Bos taurus autosome.

*Significant chromosome-wise $(P<0.05)$; **significant chromosome-wise $(P<0.01)$.

${ }^{\mathrm{G}}$ Significant genome-wise $(P<0.05)$. 
Table 12. Significant genome- and chromosome-wise single nucleotide polymorphisms (SNP) with stature

\begin{tabular}{|c|c|c|c|c|c|c|c|c|}
\hline SNP $\mathrm{NCBI}^{1}$ ID & $\mathrm{BTA}^{2}$ & Position (bp) & Heterozygosity & F-test & Effects & $P$-value & Functional class & Gene name \\
\hline rs41581655 & 1 & $95,732,116$ & 0.38 & 11.66 & -1.42 & $0.00036^{*}$ & coding-synonymous & LOC506307 \\
\hline rs41572038 & 3 & $57,607,378$ & 0.27 & 13.98 & 1.79 & $0.0001 * *$ & intron & LEC2 \\
\hline rs29011323 & 4 & $56,130,854$ & 0.48 & 11.61 & -1.26 & $0.00037^{*}$ & intron & CDC10 \\
\hline rs41592968 & 5 & $10,289,798$ & 0.24 & 13.72 & 1.89 & $0.00012^{* *}$ & intron & C3F \\
\hline rs29014633 & 5 & $74,016,631$ & 0.44 & 11.44 & 1.33 & $0.00041^{*}$ & intron & CACNG2 \\
\hline rs41591943 & 7 & $39,956,874$ & 0.37 & 12.90 & 1.51 & $0.00019 *$ & nearest_gene & LOC527275 \\
\hline rs29026038 & 11 & $63,320,525$ & 0.44 & 13.00 & 1.30 & $0.00018^{*}$ & intron & ANXA4 \\
\hline rs41640016 & 19 & $16,342,539$ & 0.17 & 11.95 & 1.97 & $0.00031^{*}$ & coding-nonsynonymous & LOC614984 \\
\hline rs41567447 & 24 & $39,886,746$ & 0.22 & 8.26 & 1.57 & $0.0022 *$ & nearest_gene & LOC781824 \\
\hline
\end{tabular}

${ }^{1} \mathrm{NCBI}=$ National Center for Biotechnology Information.

${ }^{2} \mathrm{BTA}=$ Bos taurus autosome.

*Significant chromosome wise $(P<0.05)$; **significant chromosome-wise $(P<0.01)$.

We detected 4 SNP that had significant effects on bone quality at the genome-wise and 17 at the chromosome-wise levels $(P<0.05)$ on BTA $1,3,5,7,10,11$, $13,14,19,20,21,22,23,25,26$, and 27 (Table 11). Of these 21 SNP, 13 are located in the introns of predicted genes, 6 of them are synonymous coding SNP, whereas 2 of them are not located in genes (Table 11). Five of these regions $(10,335,915 ; 12,077,407 ; 51,741,640$; $5,098,037 ; 15,147,925 \mathrm{bp}$ ) detected in the present study on BTA $7,20,23$, and 26 have been reported in a previous study (Ashwell et al., 2005). The results of current and previous studies shown are strong evidence of QTL on BTA 7, 20, 23, and 26 for bone quality in dairy cattle.

Nine SNP have been detected that have a significant effect on stature at the chromosome-wise level $(P<0.05)$ on BTA $1,3,4,5,7,11,19$, and 24 (Table 12). Two of these SNP (rs41572038 and rs41592968) on BTA 3 and BTA 5 had a significant effect on stature at the chromosome-wise level of $P<0.01$. Among the 9 significant SNP, 5 of them are located in the introns of predicted genes, and 2 of them are synonymous coding SNP (Table
12). Three of these regions $(56,130,854 ; 16,342,539$; $39,886,746 \mathrm{bp}$ ) on BTA 4,19 , and 24 have been reported by Ashwell et al. (2005). These 9 regions of the bovine genome detected in this study should serve as candidate regions for further investigation. It has been found that SNP rs41591943 shows significant association with dairy strength, and SNP rs29014633 shows significant association with 2 other traits, dairy strength and angularity.

For angularity, 5 SNP were found to have a significant effect at the genome-wise and 8 at the chromosomewise level $(P<0.05)$ on BTA 3, 5, 7, 13, 19, 26, 28, and 29 (Table 13). Of these significant SNP, 9 and 3 of them are located in the introns of predicted genes and synonymous coding SNP, respectively, and 1 of them is not located in a gene (Table 13). None of the 13 significant regions have been previously associated with angularity in dairy cattle. In addition, SNP rs41586983 also shows significant association with maternal calving ease.

Table 13. Significant genome- and chromosome-wise single nucleotide polymorphisms (SNP) with angularity

\begin{tabular}{|c|c|c|c|c|c|c|c|c|}
\hline SNP NCBI ${ }^{1}$ ID & $\mathrm{BTA}^{2}$ & Position (bp) & Heterozygosity & F-test & Effects & $P$-value & Functional class & Gene name \\
\hline rs41601701 & 3 & $98,500,067$ & 0.08 & 14.24 & 2.94 & $0.000093 * * \mathrm{G}$ & coding-synonymous & MGC139728 \\
\hline rs41604534 & 5 & $29,067,052$ & 0.55 & 8.36 & 1.03 & $0.0021 *$ & intron & KRT5 \\
\hline rs29014633 & 5 & $74,016,631$ & 0.44 & 10.37 & 1.20 & $0.00073^{*}$ & intron & CACNG2 \\
\hline rs41590827 & 5 & $75,905,624$ & 0.42 & 11.47 & 1.35 & $0.0004^{*}$ & intron & $\mathrm{RAC} 2$ \\
\hline rs41591892 & 5 & $81,801,975$ & 0.52 & 7.58 & -0.89 & $0.0033^{*}$ & intron & ITPR2 \\
\hline rs41578761 & 7 & $36,718,783$ & 0.49 & 14.82 & -1.31 & $0.000069 * * \mathrm{G}$ & intron & LOC529633 \\
\hline rs41609478 & 7 & $37,343,116$ & 0.50 & 15.77 & 1.39 & $0.000042 * * \mathrm{G}$ & nearest_gene & LOC538639 \\
\hline rs41661265 & 7 & $50,312,323$ & 0.46 & 15.46 & -1.29 & $0.000049 * * \mathrm{G}$ & coding-synonymous & MGC155209 \\
\hline rs41566192 & 13 & $43,058,415$ & 0.52 & 15.03 & -1.30 & $0.000062 * * \mathrm{G}$ & coding-nonsynonymous & MGC127374 \\
\hline rs41602212 & 19 & $37,837,691$ & 0.46 & 12.20 & 1.26 & $0.00027^{*}$ & intron & CACNA1G \\
\hline rs41648723 & 26 & $40,422,508$ & 0.23 & 9.53 & -1.45 & $0.0011^{*}$ & intron & CTBP2 \\
\hline rs41606880 & 28 & $21,540,955$ & 0.57 & 10.90 & -1.11 & $0.00054^{*}$ & intron & JDP1 \\
\hline rs41586983 & 29 & $7,579,883$ & 0.23 & 10.59 & 1.66 & $0.00064 *$ & intron & EED \\
\hline
\end{tabular}

${ }^{1} \mathrm{NCBI}=$ National Center for Biotechnology Information.

${ }^{2} \mathrm{BTA}=$ Bos taurus autosome.

*Significant chromosome-wise $(P<0.05)$; **significant chromosome-wise $(P<0.01)$.

${ }^{\mathrm{G}}$ Significant genome-wise $(P<0.05)$. 
Table 14. Significant genome- and chromosome-wise single nucleotide polymorphisms (SNP) with herd life

\begin{tabular}{|c|c|c|c|c|c|c|c|c|}
\hline SNP NCBI ${ }^{1}$ ID & $\mathrm{BTA}^{2}$ & Position (bp) & Heterozygosity & F-test & Effects & $P$-value & Functional class & Gene name \\
\hline rs29012920 & 2 & $3,605,592$ & 0.43 & 11.07 & -0.06 & $0.00049^{*}$ & intron & PROC \\
\hline rs41572038 & 3 & $57,607,378$ & 0.27 & 13.74 & 0.09 & $0.00012^{* * \mathrm{G}}$ & intron & LEC2 \\
\hline rs29013620 & 7 & $98,895,573$ & 0.33 & 15.98 & 0.09 & $0.000038 * * \mathrm{G}$ & coding-synonymous & LOC521539 \\
\hline rs41656367 & 9 & $85,942,784$ & 0.50 & 9.72 & 0.05 & $0.001^{*}$ & intron & VIL2 \\
\hline rs41658400 & 11 & $24,918,752$ & 0.50 & 8.77 & 0.05 & $0.0017^{*}$ & intron & EPAS1 \\
\hline rs41660431 & 11 & $29,041,552$ & 0.44 & 10.50 & 0.06 & $0.00068^{*}$ & intron & PPM1B \\
\hline rs41640212 & 20 & $36,431,884$ & 0.40 & 8.55 & 0.05 & $0.0019^{*}$ & intron & SLC1A3 \\
\hline rs41581582 & 20 & $51,741,640$ & 0.20 & 5.26 & 0.06 & $0.012 *$ & intron & FLJ20152 \\
\hline rs41584559 & 22 & $34,688,462$ & 0.11 & 11.11 & 0.13 & $0.00048^{* *}$ & coding-synonymous & LOC780993 \\
\hline rs41643866 & 22 & $40,066,742$ & 0.20 & 13.94 & -0.11 & $0.00011^{* * \mathrm{G}}$ & intron & LOC613533 \\
\hline rs29024274 & 22 & $46,425,659$ & 0.31 & 18.80 & -0.10 & $0.000009 * * \mathrm{G}$ & intron & CACNA1D \\
\hline rs41641232 & 23 & $19,093,160$ & 0.44 & 9.87 & -0.06 & $0.00095^{*}$ & intron & RHAG \\
\hline
\end{tabular}

${ }^{1} \mathrm{NCBI}=$ National Center for Biotechnology Information.

${ }^{2} \mathrm{BTA}=$ Bos taurus autosome.

*Significant chromosome-wise $(P<0.05)$; **significant chromosome-wise $(P<0.01)$.

${ }^{\mathrm{G}}$ Significant genome-wise $(P<0.05)$.

\section{Functional Traits}

The whole genome scan to map QTL for functional traits identified 8 SNP significantly associated with these traits at the genome-wise level and 58 SNP at the chromosome-wise level $(P<0.05)$. The details of the SNP showing association with these traits are given in Tables 14 to 19 for herd life, daughter fertility, milking speed, milking temperament, calving ease, and maternal calving ease, respectively.

Four SNP were detected to have a significant effect on herd life at the genome-wise and 12 at the chromosomewise level $(P<0.05)$ on BTA $2,3,7,9,11,12,20,22$, and 23 (Table 14). One of these regions $(19,093,160 \mathrm{bp})$ on BTA 23 has been reported by Kuhn et al. (2003) at $24 \pm 16 \mathrm{cM}$ on this chromosome, whereas the remaining regions are new findings of the present study. The confirmed QTL on BTA 23 in the current study provides strong evidence for QTL affecting herd life, and the new findings for this trait in the current paper should provide a foundation for further investigations for the QTL affecting this trait. Twelve of these significant SNP are located in the introns of predicted genes, and 4 of them are synonymous coding SNP (Table 14). It has been found that SNP rs29013620 showed significant

Table 15. Significant genome- and chromosome-wise single nucleotide polymorphisms (SNP) with daughter fertility

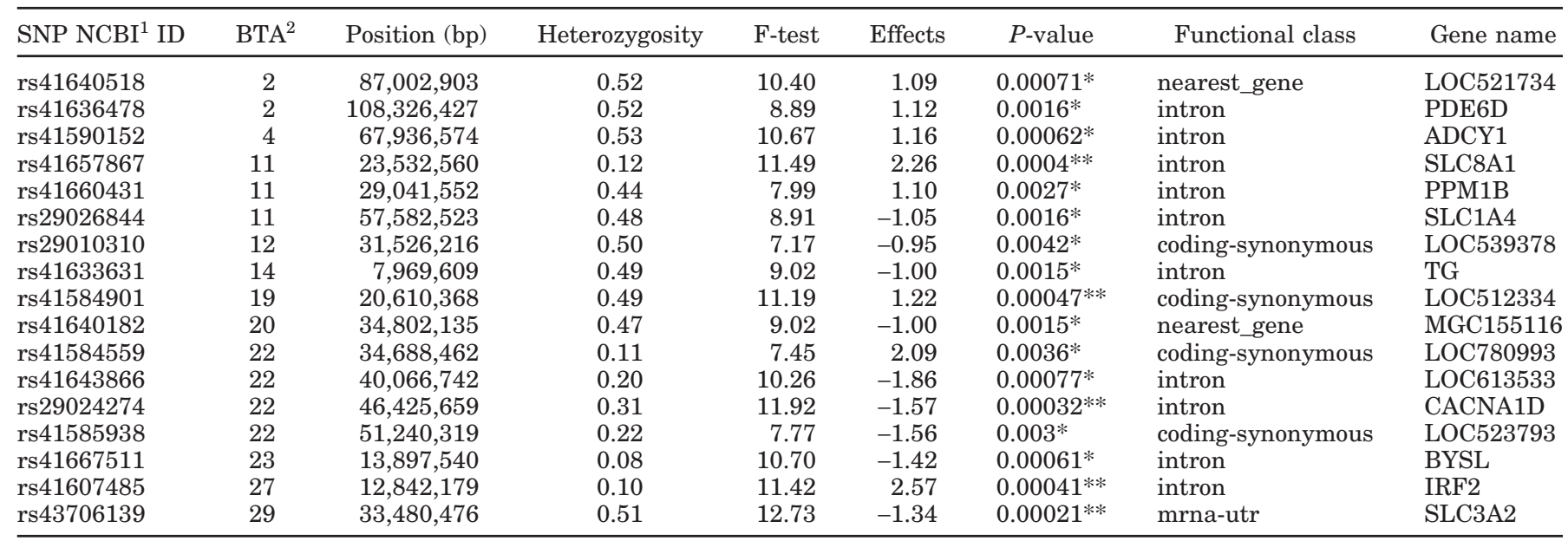

${ }^{1} \mathrm{NCBI}=$ National Center for Biotechnology Information.

${ }^{2} \mathrm{BTA}=$ Bos taurus autosome.

*Significant chromosome-wise $(P<0.05)$; **significant chromosome-wise $(P<0.01)$. 
Table 16. Significant genome- and chromosome-wise single nucleotide polymorphisms (SNP) with milking speed

\begin{tabular}{|c|c|c|c|c|c|c|c|c|}
\hline SNP NCBI ${ }^{1}$ ID & BTA $^{2}$ & Position (bp) & Heterozygosity & F-test & Effects & $P$-value & Functional class & Gene name \\
\hline rs29024165 & 1 & $8,164,917$ & 0.08 & 11.33 & 3.58 & $0.00043^{*}$ & intron & ADAMTS5 \\
\hline rs41571923 & 1 & $134,451,951$ & 0.47 & 12.38 & -1.53 & $0.00025^{* *}$ & nearest_gene & LSS \\
\hline rs29020989 & 10 & $19,944,056$ & 0.30 & 12.72 & 1.98 & $0.00021^{* *}$ & intron & TGM1 \\
\hline rs41600178 & 19 & $41,647,314$ & 0.13 & 10.57 & -2.50 & $0.00065^{*}$ & nearest gene & GAS \\
\hline rs41644418 & 24 & $20,051,012$ & 0.43 & 11.91 & 1.62 & $0.00032 * *$ & intron & HMCS \\
\hline rs41606777 & 26 & $34,495,734$ & 0.40 & 9.41 & 1.50 & $0.0012 *$ & intron & SLC18A2 \\
\hline rs41647383 & 27 & $32,834,272$ & 0.20 & 8.02 & -1.88 & $0.0026^{*}$ & coding-synonymous & FGFR1 \\
\hline
\end{tabular}

${ }^{1} \mathrm{NCBI}=$ National Center for Biotechnology Information.

${ }^{2} \mathrm{BTA}=$ Bos taurus autosome.

*Significant chromosome-wise $(P<0.05)$; **significant chromosome-wise $(P<0.01)$.

association with calving ease, and SNP rs41584559 showed significant association with daughter fertility.

For daughter fertility, 17 SNP were found to have significant effects on this trait at the chromosome-wise level $(P<0.05)$ on BTA $2,4,11,12,14,19,20,22,23$, 27, and 29 (Table 15). Five of these SNP (rs41657867, rs41584901, rs29024274, rs41607485, rs43706139) on BTA 11, 19, 22, 27, and 29 had significant effects on daughter fertility at the chromosome-wise level $(P<$ 0.01). Ten of these SNP are located in the introns of predicted genes, 4 are synonymous coding SNP, and 3 are not located in genes (Table 15). Two of these regions $(7,969,609 ; 12,842,179 \mathrm{bp})$ on BTA 14 and BTA 27 have been reported previously by Schnabel et al. (2005). Two of these SNP (rs41590152, rs41657867) also show significant association with calving ease. It has been found that SNP rs29024274 also shows significant association with milking temperament, whereas SNP rs41667511 also shows significant association with 3 more traits, maternal calving ease, calving ease, and milking temperament.

Seven SNP with significant effects on milking speed at the chromosome-wise level $(P<0.05)$ were detected on BTA $1,10,19,24,26$, and 27 (Table 16), and 3 of these SNP (rs41571923, rs29020989, rs41644418) on BTA 1,10 , and 24 had significant effects on milking speed at the chromosome-wise level $(P<0.01)$. Of these 7 significant SNP, 4 are located in the introns of predicted genes, 1 is a synonymous coding SNP, whereas 2 are not located in genes. The SNP rs41600178 on BTA $19(41,647,314 \mathrm{bp})$ has been reported to affect milking speed by Schrooten et al. (2004) at $70 \pm 15 \mathrm{cM}$, whereas the remaining 6 SNP are specific to the present study. In addition, SNP rs41606777 showed significant association with milking temperament.

One significant SNP affecting milking temperament at the genome-wise level $(P<0.05)$ and 9 SNP with a significant effect on milking temperament at the chromosome-wise level $(P<0.05)$ were detected on BTA 4 , $13,19,22,23,26$, and 29 (Table 17). It has been found that SNP rs41602212 on BTA 19, located at 37,837,691 $\mathrm{bp}$, has an F-test value of 21.67 and allele substitution effects of 2.11. Six of these significant SNP are located in the introns of predicted genes, 3 of them are synonymous, and 1 of them is not located in a gene region (Table 17).

Two SNP had a genome-wise and 7 SNP had a chromosome-wise level of significant effects $(P<0.05)$ on

Table 17. Significant genome- and chromosome-wise single nucleotide polymorphisms (SNP) with milking temperament

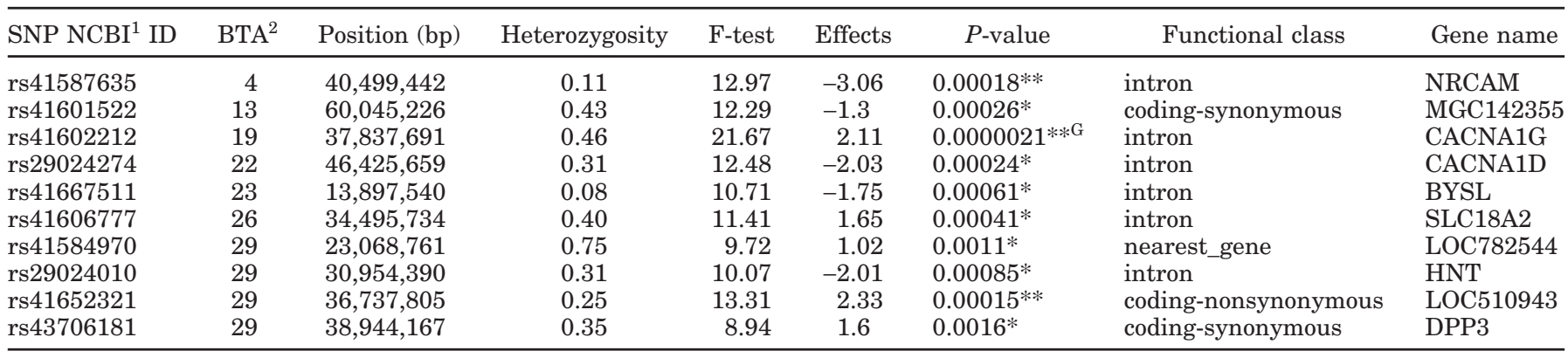

${ }^{1} \mathrm{NCBI}=$ National Center for Biotechnology Information.

${ }^{2} \mathrm{BTA}=$ Bos taurus autosome.

*Significant chromosome-wise $(P<0.05)$; **significant chromosome-wise $(P<0.01)$.

${ }^{\mathrm{G}}$ Significant genome-wise $(P<0.05)$. 
Table 18. Significant genome- and chromosome-wise single nucleotide polymorphisms (SNP) with direct calving ease

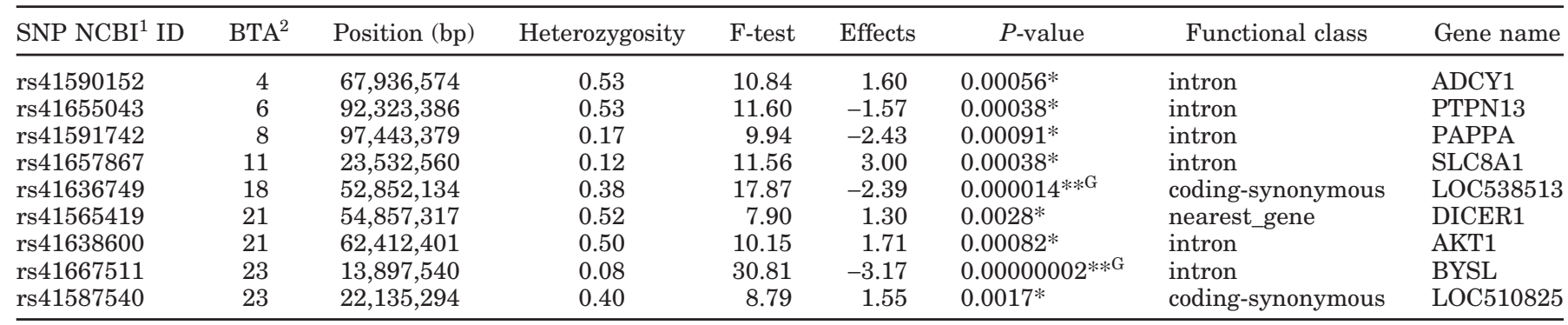

${ }^{1} \mathrm{NCBI}=$ National Center for Biotechnology Information.

${ }^{2} \mathrm{BTA}=$ Bos taurus autosome.

*Significant chromosome-wise $(P<0.05)$; **significant chromosome-wise $(P<0.01)$.

${ }^{\mathrm{G}}$ Significant genome-wise $(P<0.05)$.

direct calving ease on BTA $4,6,8,11,18,21$, and 23 (Table 18). Six of these significant SNP are located in the introns of predicted genes, and 2 of them are synonymous coding SNP (Table 18). Four of these regions $(92,323,386 ; 97,443,379 ; 13,897,540 ; 22,135,294 \mathrm{bp})$ on BTA 6,8 , and 23 were previously associated with direct calving ease by Smaragdov et al. (2006).

One and 6 SNP had significant effects on maternal calving ease at the genome- and chromosome-wise levels $(P<0.05)$, respectively, on BTA 7, 9, 23, 24, 28, and 29. Of the 7 significant SNP, 5 are located in the introns of predicted genes, and 2 of them are synonymous coding SNP (Table 19). Two of these regions $(85,942,784$; 41,818,493 bp) on BTA 9 and BTA 24 were previously associated with maternal calving ease by Ashwell et al. (2005).

\section{CONCLUSIONS}

This study has used a medium-density genome-wide scan, using SNP markers located in or nearby known genes. Considering the marker density used, it is unlikely that any of the SNP found associated with conformational or functional traits represent causal muta- tions. It does, however, provide a starting point for uncovering quantitative trait nucleotides, particularly where the results of this study are backed up by other independent studies. Among the 60 significant SNP for the 5 major type traits, 5 of these regions have been reported in previous studies by various authors. Of the 70 SNP that had significant associations with 6 descriptive type traits, 12 of the regions have been reported in previous studies. Of the 66 SNP associated with functional traits, 10 of these regions have been reported in previous studies. Use of much denser marker sets recently developed for cattle will no doubt aid in moving from the marker associations, such as those reported in this study, toward revealing the causal mutations underlying many of the economically important traits in dairy cattle.

\section{ACKNOWLEDGMENTS}

We acknowledge the financial contributions of Semex Alliance (Guelph, Ontario, Canada), the Alberta Livestock Industry Development Fund (Edmonton, Alberta, Canada), Institute for Food and Agricultural Science Alberta (Edmonton, Alberta, Canada), and Natural Sci-

Table 19. Significant genome- and chromosome-wise single nucleotide polymorphisms (SNP) with maternal calving ease

\begin{tabular}{|c|c|c|c|c|c|c|c|c|}
\hline $\mathrm{SNP}_{\mathrm{NCBI}}{ }^{1} \mathrm{ID}$ & BTA $^{2}$ & Position (bp) & Heterozygosity & F-test & Effects & $P$-value & Functional class & Gene name \\
\hline rs29013620 & 7 & $98,895,573$ & 0.33 & 16.21 & 2.49 & $0.000034 * * \mathrm{G}$ & coding-synonymous & LOC521539 \\
\hline rs41656367 & 9 & $85,942,784$ & 0.50 & 9.10 & 1.46 & $0.0014^{*}$ & intron & VIL2 \\
\hline rs41667511 & 23 & $13,897,540$ & 0.08 & 12.52 & -2.2 & $0.00023^{*}$ & intron & BYSL \\
\hline rs29023262 & 28 & $11,084,209$ & 0.52 & 7.60 & -1.37 & $0.0033^{*}$ & intron & CHRM3 \\
\hline rs41652496 & 28 & $23,732,208$ & 0.33 & 9.22 & 1.77 & $0.0013^{*}$ & intron & $\mathrm{PP}$ \\
\hline rs41586984 & 29 & $7,578,139$ & 0.39 & 9.09 & 1.78 & $0.0014^{*}$ & intron & EED \\
\hline
\end{tabular}

${ }^{1} \mathrm{NCBI}=$ National Center for Biotechnology Information.

${ }^{2} \mathrm{BTA}=$ Bos taurus autosome.

*Significant chromosome-wise $(P<0.05)$; **significant chromosome-wise $(P<0.01)$.

${ }^{\mathrm{G}}$ Significant genome-wise $(P<0.05)$. 
ences and Engineering Research Council of Canada (Ottawa, Ontario).

\section{REFERENCES}

Ashwell, M. S., D. W. Heyen, J. I. Weller, T. S. Soenstegaard, C. P. Van Tassell, and H. A. Lewin. 2005. Detection of quantitative trait loci influencing conformation traits and calving ease in HolsteinFriesian cattle. J. Dairy Sci. 88:4111-4119.

Benjamini, Y., and Y. Hochberg. 1995. Controlling the false discovery rate: A practical and powerful approach to multiple testing. J. R. Stat. Soc. Ser. B. Methodol. 57:289-300.

Boichard, D., C. Grohs, F. Bourgeois, F. Cerqueira, R. Faugeras, A. Neau, R. Rupp, Y. Amigues, M. Y. Boscher, and H. Leveziel. 2003. Detection of genes influencing economic traits in three French dairy cattle breeds. Genet. Sel. Evol. 35:77-101.

Daw, E. W., S. C. Heath, and Y. Lu. 2005. Single-nucleotide polymorphism versus microsatellite markers in a combined linkage and segregating analysis of a quantitative trait. BMC Genet. 6(Suppl 1):S32.

Dekkers, J. C. M., and F. Hospital. 2002. The use of molecular genetics in the improvement of agricultural populations. Nat. Rev. Genet. 3:22-32.

Farnir F., W. Coppieters, J. J. Arranz, P. Berzi, N. Cambisano, B. Grisart, L. Karim, F. Marcq, L. Moreau, M. Mni, C. Nezer, P. Simon, B. Vanmanshoven, D. Wagenaar, and M. Georges. 2000. Extensive genome-wide linkage disequilibrium in cattle. Genome Res. 10:220-227.

Fernando, R. L., and M. Grossman. 1989. Marker assisted selection using best linear unbiased prediction. Genet. Sel. Evol. 21:467-477.

Fernando, R. L., D. Nettleton, B. R. Southey, J. M. C. Dekkers, M. F. Rothschild, and M. Soller. 2004. Controlling the proportion of false positive in multiple dependent tests. Genetics 166:611-619.

Gautier, M., R. R. Barcelona, S. Fritz, C. Grohs, T. Druet, D. Boichard, A. Eggen, and T. H. E. Meuwisen. 2006. Fine mapping and physical characterization of two linked QTLs affecting milk fat. Genetics 172:425-436.

George, A. W., P. M. Visscher, and C. S. Haley. 2000. Mapping quantitative trait loci in complex pedigrees. Genetics 156:2081-2092.

Georges, M., D. Nielsen, M. Mackinnon, A. Mishra, R. Okimoto, A. T. Pasquino, L. S. Sargeant, A. Soerensen, M. R. Steele, X. Zhao, J. E. Womack, and I. Hoeschele. 1995. Mapping QTL controlling milk production in dairy cattle by exploiting progeny testing. Genetics 139:907-920.

Gilmour, A. R., B. J. Gogel, B. R. Cullis, and R. Thompson. 2006. ASReml User Guide Release 2.0. VSN Int. Ltd, Hemel Hempstead, UK.

Grapes, L., J. C. M. Dekkers, M. F. Rothschild, and R. L. Fernando. 2004. Comparing linkage disequilibrium-based methods for fine mapping quantitative trait loci. Genetics 166:1561-1570.

Hinds, D. A., L. L. Stuve, G. B. Nilsen, E. Halperin, E. Eskin, D. G. Ballinger, K. A. Frazer, and D. R. Cox. 2005. Whole-genome patterns of common DNA variation in three human populations. Science 307:1072-1079.

Kolbehdari, D., G. B. Jansen, L. R. Schaeffer, and O. B. Allen. 2005. Power of QTL detection by either fixed or random models in halfsib designs. Genet. Sel. Evol. 37:601-614.

Kuhn, C., J. Bennewitz, N. Reinsch, N. Xu, H. Thomsen, C. Looft, G. A. Brockmann, M. Schwerin, C. Weimann, S. Hiendleder, G. Erhardt, I. Medjugorac, M. Forster, B. Brenig, F. Reinhardt, R. Reents, I. Russ, G. Averdunk, J. Blumel, and E. Kalm. 2003. QTL mapping of functional traits in the German Holstein cattle population. J. Dairy Sci. 86:360-368.

Meuwissen, T. H. E., and M. E. Goddard. 2000. Fine mapping of quantitative trait loci using linkage disequilibria with closely linked markers. Genetics 155:421-430.

Oliphant, A., D. L. Barker, J. R. Stuelpnagel, and M. S. Chee. 2002 BeadArray technology: Enabling an accurate, cost-effective approach to high-throughput genotyping. Biotechniques Suppl. $56-58$.

Schnabel, R. D., T. S. SoEnstega Ard, J. F. Taylor, and M. S. Ashwel. 2005. Whole genome scan to detect QTL for milk production, conformation, fertility and functional traits in two US Holstein families. Anim. Genet. 36:408-416.

Schrooten, C., M. C. A. M. Bink, and H. Bovenhuis. 2004. Whole genome scan to detect chromosomal regions affecting multiple traits in dairy cattle. J. Dairy Sci. 87:3550-3560.

Smaragdov, M. G., E. M. Prinzenberg, and L. Zwierzchowski. 2006. QTL mapping in cattle: Theoretical and empirical approach. Anim. Sci. Pap. Rep. 24:69-110.

Snelling, W. M., E. Casas, R. T. Stone, J. W. Keele, G. P. Harhay, G. L. Bennet, and T. P. Smith. 2005. Linkage mapping bovine EST-based SNP. BMC Genomics 6:74.

Zhao, H. H., R. L. Fernando, and J. C. M. Dekkers. 2007. Power and precision of alternate methods for linkage disequilibrium mapping of quantitative trait loci. Genetics 175:1975-1986. 